\title{
Schweizerische Gesellschaft für Nephrologie (SGN-SSN)

\section{Isabelle Binet}

Präsidentin, für den Vorstand der SGN-SSN
Die offizielle Webseite der SGN finden Sie unter www.nephro.ch. Das Menü gibt Zugang zu Vorstand Kommissionen und Arbeitsgruppen, zu einer Adressliste der Dialysenzentren und zu edukativen Links und kommenden Veranstaltungen. Auch der Jahreskongress präsentiert sich auf der Homepage; der nächste Kongress findet vom 2. bis 4 . Dezember 2009 in Interlaken statt.
Die Schweizerische Gesellschaft für Nephrologie (SGN-SSN) wurde 1969 in Bern von 17 Mitgliedern gegründet. Nun zählt die SGN 220 Mitglieder, davon praktizieren 160 als Fachärzte für Nephrologie. Die SGN ist Mitglied der FMH.

Im Vorstand, bestehend aus neun Mitgliedern, sind die italienisch-, französisch- und deutschsprechende Schweiz wie auch die universitären und nichtuniversitären Zentren, die niedergelassenen Kollegen, die Nierenphysiologie und die Nephropädiatrie vertreten.

\section{Aufgaben und Ziele}

Neben dem Hauptevent einer wissenschaftlichen Jahresversammlung, die meistens im Dezember stattfindet, ist die SGN mit verschiedenen Kommissionen verantwortlich für die vielfältigen Tätigkeiten der Schweizer Nephrologen. Die Weiter- und Fortbildungskommission bestimmt den 3-jährigen, spezifisch nephrologischen Teil des Weiterbildungsprogramms zum Facharzt in Nephrologie sowie den Inhalt des Fortbildungsprogramms. Das Programm zum Schwerpunkt in pädiatrischer Nephrologie wird durch eine pädiatrische Arbeitsgruppe bestimmt. Die Dialysekommission kümmert sich um alle Belange der Dialysebehandlung sowohl bei Kindern wie Erwachsenen und erarbeitet insbesondere deren Tarife in Zusammenarbeit mit den Krankenkassen. Die Ultraschallkommission organisiert Kurse für die Ultraschallausbildung der Nephrologen. Verschiedene Arbeitsgruppen befassen sich mit dem nationalen Register für Patienten mit Nierenersatztherapie, mit nephrologischer Pflege, mit Einsätzen bei humanitären Katastrophen wie Kriegen und Erdbeben u.a. Wichtig ist auch die schweizerische Nierenstiftung (www.nierenstiftung.ch), in deren Stiftungsrat die SGN mit verschiedenen Mitgliedern vertreten ist.

Kontakte mit anderen Gesellschaften sind ein zentrales Interesse der SGN. Eine kollektive Mitgliedschaft in der International Society of
Nephrology (ISN) existiert seit 2005. In Abständen finden gemeinsame Tagungen mit der französischen oder deutschen Gesellschaft für Nephrologie oder wie am kommenden 24.-26. September als Gastgesellschaft bei der Schweizerischen Gesellschaft für Intensivmedizin in St. Gallen statt.

Die Plattform für die Präsentation von laufenden wissenschaftlichen Projekten aus der Schweiz ist der Jahreskongress im Dezember.

Seit drei Jahren ist der Tag «Basics in Nephrology» in der nephrologischen Fortbildung institutionalisiert. Der Jahreskongress vermittelt auch vermehrt Information über moderne Pathophysiologie und Pathologie. Unter der Leitung der Ultraschallskommission wurde 2008 ein Kurs für die Ultraschalluntersuchung von Niere, Harnwegen und Dialyseshunts mit grossem Erfolg eingeführt.

Seit dem Erdbeben 1988 entwickelte sich eine Zusammenarbeit mit Armenien unter der Leitung der Arbeitsgruppe Schweiz-Armenien. Über 20 Jahre gab es regelmässigen Austausch und eine kontinuierliche Weiterbildungsunterstützung für Nephrologen in Armenien. Für Katastropheneinsätze und die Entwicklung der Nephrologie in anderen Ländern wird nun die humanitäre Kommission ein Konzept entwickeln und zusammen mit der internationalen Gesellschaft für Nephrologie erarbeiten.

\section{Niereninsuffizienz vorbeugen}

Weitere Ziele sind die Vertiefung der Kontakte und gemeinsame Projekte mit der schweizerischen Nierenstiftung. Angesichts der zunehmenden Prävalenz nierenkranker Patienten sieht es die SGN als wichtige Aufgabe, die Wahrnehmung und die Prävention der chronischen Niereninsuffizienz in der Bevölkerung anzugehen. In diesen Rahmen werden Projekte zum jährlichen «World Kidney Day» in Partnerschaft unterstützt (www.worldkidneyday.org). 